\title{
Size distributions of misrejoining DNA fragments in irradiated cells
}

\author{
Tomas Radivoyevitch a,*, David G. Hoel a, \\ Philip Hahnfeldt ${ }^{\mathrm{b}}$, Rainer K. Sachs ${ }^{\mathrm{c}}$ \\ a Department of Biometry and Epidemiology, Medical University of South Carolina, Charleston, SC \\ 29425, USA \\ b Joint Center for Radiation Therapy, Harvard Medical School, Boston, MA 02115, USA \\ ${ }^{\mathrm{c}}$ Departments of Mathematics and Physics, University of California, Berkeley, CA 94720, USA
}

Received 24 June 1997; received in revised form 9 January 1998

\begin{abstract}
When ionizing radiation strikes a cell it induces DNA double strand breaks (DSBs). Subsequently, some of the DSBs misrejoin and thus cause alterations in the size distribution of the DNA fragments. We derive a system of non-linear integro-differential equations describing the misrejoining interactions of five classes of DNA fragments, including rings and various types of linear fragments. The fragment classes are represented by density functions; the shape of a density function determines the probability that a fragment has a particular size and the amplitude (integral) equals the expected number of such fragments per cell. The equations are solved: analytically for exponentially distributed initial fragment sizes (corresponding to high doses) and numerically for arbitrary initial conditions. Computed final fragment size distributions are applied to situations representative of flow karyotypes and pulsed-field gel assays. For human flow karyotypes, the model can be used to obtain misrejoining estimates at doses too high for conventional methods of data analysis. For pulsed-field gel assays in which human chromosomes are digested with restriction endonucleases to form 'cut-somes' (restriction fragments), the model provides a means of misrejoining estimation when the cut-some sizes are non-random. The model suggests that if the cut-some size distribution for unirradiated cells is completely random, misrejoining of radiation-induced DSBs will not be detectable in the final size distribution. (C) 1998 Elsevier Science Inc. All rights reserved.
\end{abstract}

\footnotetext{
${ }^{*}$ Corresponding author. Tel.: 1-803 766 7064; fax: 1-803 876 1126; e-mail: radivot@musc.edu.
} 
Keywords: Double-strand break; SSEB model; DNA fragment sizes; Misrejoining

\section{Introduction}

Ionizing radiation induces DNA double strand breaks (DSBs) ${ }^{1}$ which can subsequently misrejoin within a cell. Since DSB misrejoining is believed to play a key role in radiation carcinogenesis [1], characterizing the mechanisms of DSB misrejoining is an important aspect of radiation risk assessment.

Misrejoining leads to reshuffling of chromosome fragments, thus altering their sizes. In a typical approach to misrejoining estimation [2], measured DNA fragment size distributions are converted to a total quantity (e.g. the fraction of fragments outside a band of specific sizes) which is then further converted to misrejoinings. To improve such estimates, models that relate misrejoinings to a theoretical fragment size distribution are needed so that distribution-based (least squares or maximum likelihood) estimates can be made directly from the data, thus increasing the amount of measured distribution shape information transferred to the estimates. Although induced (i.e. initial) DNA fragment size distribution models have been developed [3-7] and used to form distribution-based DSB estimates immediately following an acute exposure [8,9], no misrejoining estimates based on theoretical size distributions during and after misrejoining have yet been published. We here present a model of the size distribution dynamics of misrejoining DNA fragments. The model can lead to distribution-based estimates of the final number of misrejoinings using data from: (1) pulsed-field gel electrophoresis (PFGE) applied to misrejoined human chromosomes that have been cut with restriction enzymes immediately before measurement; or (2) flow karyotypes following irradiation at doses greater than 10 Gy (conventional methods [10] can be applied at lower doses).

We shall first review the standard model for size distributions just after an acute dose of low LET (i.e. sparsely ionizing) radiation. Then we will introduce a set of non-linear integro-differential equations that represent the temporal evolution of sizes for misrejoining chromosomes. Densities are used to describe size distributions of rings and of linear fragments with two, one or no reactive ends. Despite the non-linearity of the equations, we show that the time development can be explicitly integrated by techniques involving Fourier transforms of the densities. The relevance of final size distributions (i.e. after misrejoining

\footnotetext{
1 Abbreviations: DSB = double-strand break; $\mathrm{EtBr}=$ ethidium bromide; LET = linear energy transfer; PFGE = pulsed-field gel electrophoresis; $\mathrm{SSEB}=$ Sax subset ethidium bromide; FFT = fast Fourier transform; PRA = peak reduction algorithm; BNA = background number algorithm.
} 
has run its full course) to flow karyotype data and PFGE measurements are then discussed.

\section{Background}

\subsection{DSB misrejoining}

In an unirradiated human cell during the $G_{0} / G_{1}$ part of the cell cycle, there are 46 chromosomes. Each chromosome has a telomere at each of its two ends. The chromosomes vary in size, from about $60 \mathrm{Mb}\left(1 \mathrm{Mb}=10^{6}\right.$ base pairs $)$ to almost $300 \mathrm{Mb}$. After an acute dose of ionizing radiation, double-strand breaks (DSBs) cut the chromosomes into DNA fragments of various sizes. Each DSB has two free ends. In a breakage-and-reunion scenario, similar to that originally suggested by Sax [11] and others, a free end can undergo binary misrejoining by pairing up with a free end from a different DSB. The result is a reshuffling of chromosomes that entails changes in the pattern of chromosome sizes. During this misrejoining process the telomeres are inert.

It is known that the majority of DSBs induced by radiation do not participate in misrejoining. Rather, the two free ends of a DSB 'eurejoin', i.e. rejoin with each other to restore the original DNA sequence, or at most cause a local alteration too small to show up in the size distribution assays of interest here. We shall here refer to the DSBs that do participate in misrejoining as 'reactive', i.e., in the terminology of Radivoyevitch et al. [12], reactive DSBs are active DSBs which do not undergo accidental binary eurepair. Experimental estimates for the fraction of DSBs that are reactive, and theoretical methods for calculating this fraction as a function of radiation dose, are summarized elsewhere [7,12]. The experimental assays to be discussed in the present paper are sensitive only to misrejoinings, so that only the reactive DSBs are relevant here.

\subsection{The ethidium bromide (EtBr) model}

Fragment size distribution models are formulated in terms of fragment number densities $n_{c}(x, t)$, where $x$ is the fragment size (DNA content) and $n_{c}(x, t) \mathrm{d} x$ is the expected number of type $c$ fragments in the interval $[x, x+\mathrm{d} x]$ at time $t$. We consider five types of fragment number densities in irradiated cells:

1. $n_{a t}(x, t)$ - the number density of 'edges', i.e. of fragments in which one end is the free end of a reactive DSB while the other end is telomeric;

2. $n_{a a}(x, t)$ - the number density of 'internals', fragments both of whose ends are free ends of (different) reactive DSBs;

3. $n_{t t}(x, t)$ - the number density of 'bitelomeres', misrejoined fragments in which both ends are telomeric; 
4. $n_{r}(x, t)$ - the number density of ring fragments; and

5. $n_{\delta}(x, t)$ - the number density of 'delta' fragments, chromosomes that never contained a reactive DSB. ${ }^{2}$

Note that each of these fragment densities can be viewed as an amplitude factor $N_{c}(t)$, the expected number of type $c$ fragments, multiplied by a shape factor $p_{c}(x, t)$, the probability density that a fragment has size $x$, i.e. $n_{c}(x, t)=N_{c}(t) p_{c}(x, t)$, where $N_{c}(t)=\int_{0}^{\infty} n_{c}(x, t) \mathrm{d} x$ and $\int_{0}^{\infty} p_{c}(x, t) \mathrm{d} x=1$.

The EtBr model (for reviews see Refs. [6,7]) is a parameterization of the density functions at $t=0^{+}$, just after acute irradiation but before DSBs have had time to misrejoin; this implies $n_{t t}\left(x, 0^{+}\right)=0$ and $n_{r}\left(x, 0^{+}\right)=0$. The name 'EtBr' refers to the ethidium bromide treatment used to make DNA detectable in certain assays [6]. The EtBr model applies to low LET, i.e. to sparsely ionizing radiation such as high-energy photons, but not in general to high LET, i.e. not to densely ionizing radiation such as neutrons. We now provide a brief derivation of the model for a cell with one chromosome of size $S$. Extending the derivation to the case of $N$ chromosomes is straightforward, see Eqs. (9) and (10).

The EtBr model assumes complete randomness, i.e. that each unit length of DNA has equal probability $\lambda$ of incurring a reactive DSB, or equivalently [6], that the number of DSBs on a particular DNA stretch of length $x$ is Poisson with mean $\lambda x$. (The units of $\lambda, \mathrm{bp}^{-1}$ in theoretical sections (e.g. here) and $\mathrm{Mb}^{-1}$ in application sections (later), correspond to the inverse of the units used for fragment sizes.) This randomness assumption is meant only for averages over stretches of many thousands of base pairs, large compared to such local structures as nucleosomes but small compared to a chromosome. Moreover, the randomness assumption is not appropriate for high LET [13,14], so the EtBr model is meant primarily for low LET. Randomness together with standard properties of the Poisson distribution imply that the probability that the $x$ th base pair of a chromosome is hit (with a reactive DSB) while the base pairs between it and the 0 th base pair (telomere) are unhit is $\lambda \mathrm{e}^{-\lambda x}$. Since there are two edges per chromosome we have [6,7]

$$
n_{a t}\left(x, 0^{+}\right)=2 \lambda \mathrm{e}^{-\lambda x} u_{0}(x, S),
$$

where $u_{0}(x, S)$, equal to one on the interval $[0, S]$ and zero elsewhere, is used to indicate that an edge cannot be larger than the original chromosome of size $S$. For internal fragments, $\lambda \mathrm{e}^{-\lambda x} \lambda$ is the probability that two reactive DSBs occur at two specific sites while the $x$ base pairs between them remain untouched. As there are $S-x$ chromosomal sites from which an internal of size $x$ can arise, one gets [6]

$$
n_{a a}\left(x, 0^{+}\right)=\lambda^{2} \mathrm{e}^{-\lambda x}(S-x) u_{0}(x, S) .
$$

\footnotetext{
${ }^{2}$ Delta fragments are named after the Dirac delta function that describes their density of sizes.
} 
Finally, $\mathrm{e}^{-\lambda S}$ is the probability that no DSBs strike the chromosome and thus

$$
n_{\delta}\left(x, 0^{+}\right)=\mathrm{e}^{-\lambda S} \delta(x-S)
$$

where $\delta(x-S)$ is the Dirac delta function centered at $x=S$.

In these equations $\lambda$ is to be regarded as an input parameter whose value at any particular radiation dose must be determined either by an experimental assay of total misrejoinings after misrejoining has run its full course, or by a separate model such as the SMBE (Sax Markov binary eurejoining/misrejoining) model of Radivoyevitch et al. [12]. Recall that $\lambda$ refers only to DSBs that are reactive. DSBs which will ultimately eurejoin but are temporarily present do influence size distributions observed with PFGE at $t=0^{+}$[7], but need not and will not be considered in the following analysis.

We shall now develop a probabilistic formalism for calculating the time development of size changes after various radiation doses, and discuss comparisons of the theoretical predictions for $t=\infty$ with flow karyotype experiments. We shall show later that essentially the same formalism also applies to some other experiments involving pulsed-field gel electrophoresis (PFGE) in which restriction enzymes are used to cut the chromosomes into smaller pieces.

\section{The Sax subset EtBr (SSEB) model}

The Sax subset EtBr (SSEB) model is an extension of the EtBr model to include DSB misrejoining dynamics. In the acronymn, 'Sax' refers to the type of misrejoining (involving DSB free ends), 'subset' indicates that only reactive DSBs are considered (see above), and 'EB' (for ethidium bromide) indicates that fragments are uniformly detectable as, for example, with EtBr gel staining (sequence specific probes require different models $[5,6]$ ). The SSEB model is defined in this section and solved in subsequent sections. The model uses an approximation, essentially that ring formation is not too frequent, which will be justified retroactively when the model solutions are found.

Just after an acute dose (i.e. at $t=0^{+}$), all of the fragment density lies within the edge, internal, and delta fragment pools, i.e. $n_{r}\left(x, 0^{+}\right)=0=n_{t t}\left(x, 0^{+}\right)$. As time evolves, DNA leaves the edge and internal pools and moves into the ring and bitelomere pools. Fig. 1 depicts this process, showing that edges misrejoin with edges to form bitelomeres, edges misrejoin with internals to form bigger edges, internals misrejoin with other internals to form larger internals, and an internal can misrejoin with itself to form a ring. The delta pool remains constant. It is assumed that when the system reaches its final state (i.e. after repair and misrepair have run their full course; formally, at $t=\infty$ ) all reactive DSB free ends will have found partners, hence, the edge and internal fragment pools will be empty, the correctly repaired fragments will all lie in the delta pool, and the misrejoined fragments will be rings or bitelomeres. 


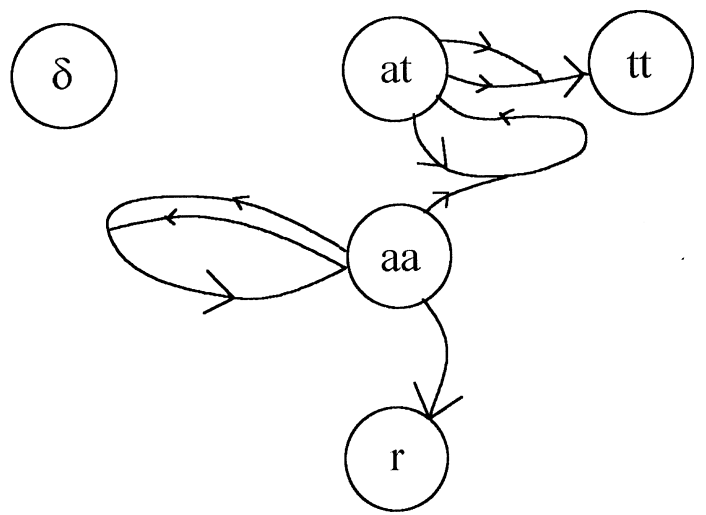

Fig. 1. This SSEB model fragment flow graph illustrates that: (1) edges merge with edges to form bitelomeres, (2) edges merge with internals to form larger edges, (3) internals merge with internals to form larger internals, and (4) internals form rings.

The dynamics that take place between the induced (i.e. initial) distribution and the final distribution will now be described, first mathematically and then by motivating descriptions. The equations of the SSEB model are the following:

$$
\begin{aligned}
\frac{\partial n_{a t}(x, t)}{\partial t}= & -\kappa n_{a t}(x, t) N_{a t}(t)-2 \kappa n_{a t}(x, t) N_{a a}(t) \\
& +2 \kappa \int_{0}^{x} n_{a t}\left(x^{\prime}, t\right) n_{a a}\left(x-x^{\prime}, t\right) \mathrm{d} x^{\prime} \\
\frac{\partial n_{a a}(x, t)}{\partial t}= & -4 \kappa n_{a a}(x, t) N_{a a}(t)-2 \kappa n_{a a}(x, t) N_{a t}(t) \\
& +2 \kappa \int_{0}^{x} n_{a a}\left(x^{\prime}, t\right) n_{a a}\left(x-x^{\prime}, t\right) \mathrm{d} x^{\prime} \\
\frac{\partial n_{t t}(x, t)}{\partial t}= & \frac{\kappa}{2} \int_{0}^{x} n_{a t}\left(x^{\prime}, t\right) n_{a t}\left(x-x^{\prime}, t\right) \mathrm{d} x^{\prime} \\
\frac{\partial n_{r}(x, t)}{\partial t}= & \kappa n_{a a}(x, t) \\
\frac{\partial n_{\delta}(x, t)}{\partial t}= & 0, \\
n_{a t}\left(x, 0^{+}\right)= & 2 \lambda \mathrm{e}^{-\lambda x} \sum_{j=1}^{N} u_{0}\left(x, S_{j}\right), \quad n_{a a}\left(x, 0^{+}\right)=\lambda^{2} \mathrm{e}^{-\lambda x} \sum_{j=1}^{N}\left(S_{j}-x\right) u_{0}\left(x, S_{j}\right) \\
n_{\delta}\left(x, 0^{+}\right)= & \sum_{j=1}^{N} \delta\left(x-S_{j}\right) \mathrm{e}^{-\lambda S_{j}}, \quad n_{r}\left(x, 0^{+}\right)=0, \quad n_{t t}\left(x, 0^{+}\right)=0
\end{aligned}
$$


where $\kappa$ is the binary misrepair rate constant for reactive DSB free ends [12], $N_{c}(t)=\int_{0}^{\infty} n_{c}(x, t) \mathrm{d} x$ is the total number of fragments in pool $c$ (where $c$ is either $a t, a a, t t, r$ or $\delta$ ), $\lambda$ is the number of reactive DSBs per base pair at $t=0^{+}$ and also the number of misrejoined DSBs per base pair at $t=\infty, S_{j}$ is the size of the $j$ th chromosome, $u_{0}\left(x, S_{j}\right)$ equals one on the interval $\left[0, S_{j}\right]$ and zero elsewhere, $\delta\left(x-S_{j}\right)$ is a Dirac delta function centered at $x=S_{j}$ and $N$ is the total number of chromosomes within the cell.

The terms of the SSEB model fall into two categories: source terms such as $-\kappa n_{a t}(x, t) N_{a t}(t)$ in Eq. (4) and target terms. The source terms are negative because they withdraw fragment density to supply the target terms which are positive. The source terms include pool size factors such as $N_{a t}(t)$ because an edge or internal of size $x$ can react with any other edge or internal. The target terms are convolution integrals that include all possible fragment interactions leading to a misrejoined fragment of size $x$, i.e. all interactions between fragments of size $x^{\prime}$ and $x-x^{\prime}$. Specific explanations for each equation are now given.

Eq. (4) describes the rate of size $x$ edge fragment formation. The first and second terms are the rates at which density leaves $n_{a t}(x, t)$ due to misrejoining reactions. It is assumed that an edge of size $x$ can react with any other edge or internal with equal probability except that internals are twice as likely since they have two reactive ends. The third term describes how density moves into $n_{a t}(x, t)$ through misrejoining events between all possible combinations of fragments of size $x^{\prime}$ and $x-x^{\prime}$. This convolution term, when integrated over all $x$, is equal in magnitude to the second term since any density lost from $n_{a t}(x, t)$ by reaction with an internal is regained by some other edge at some other $x$.

Eq. (5) describes the rate at which internal fragments of size $x$ are formed. The first two terms are the rates at which fragment density leaves $n_{a a}(x, t)$ due to misrejoining events. Because internal fragments have two reactive ends, the self-reaction rate is multiplied by a factor of 4 and the cross-reaction rate by a factor of 2. The third term is an auto-convolution integral that represents the formation of $n_{a a}(x, t)$ through misrejoining events between two smaller internal fragments. The convolution integral is multiplied by a factor of two, rather than four, because fragment interactions on the interval $x^{\prime}<\frac{1}{2} x$ are identical with those on the interval $x^{\prime}>\frac{1}{2} x$. The total flux (integral over $x$ ) of the first term equals twice the total flux of the third term since the joining of two internal fragments produces one larger internal fragment. The loss of internal fragments due to ring closure is neglected because it is a linear kinetic term that cannot compete effectively with the quadratic kinetic terms (i.e. the loss of internals to larger internals and the loss of internals to edges). This neglect will be justified later, by appropriate numerical estimates.

Eq. (6) states that bitelomeric fragments of size $x$ are synthesized by two misrejoining edges whose lengths add to $x$. The total flux (integral over $x$ ) of the auto-convolution term equals one-half the total flux of the first term in Eq. (4) since two misrejoining edges form one larger bitelomere. 
In Eq. (7), rings are synthesized at a rate equal to $\kappa$ times the density of internals. There is no change in size so there are no interactions with densities at other $x$ values. The SSEB model does not quite conserve mass since, as mentioned, the flux entering the ring pool is neglected as a flux leaving the internal pool.

The initial conditions, Eqs. (9) and (10), are those of the EtBr model discussed above. Other initial conditions can also be solved, if they are known. Note from Eq. (8) that the delta density remains at its initial condition, i.e. $n_{\delta}(x, t)=n_{\delta}\left(x, 0^{+}\right)$.

\section{SSEB pool size equations and solutions}

Remarkably, the time integrals of the SSEB integro-differential Eqs. (4)-(7) can be found. This section starts the integration process by solving for the pool sizes, i.e. for the total number $N_{a t}(t)$ of edge fragments at time $t$ and for similar quantities.

The SSEB model integrated over $x$ yields the pool size equations

$$
\begin{aligned}
& \frac{\mathrm{d} N_{a t}(t)}{\mathrm{d} t}=-\kappa N_{a t}^{2}(t), \\
& \frac{\mathrm{d} N_{a a}(t)}{\mathrm{d} t}=-2 \kappa N_{a a}^{2}(t)-2 \kappa N_{a t}(t) N_{a a}(t), \\
& \frac{\mathrm{d} N_{t t}(t)}{\mathrm{d} t}=\frac{\kappa}{2} N_{a t}^{2}(t), \\
& \frac{\mathrm{d} N_{r}(t)}{\mathrm{d} t}=\kappa N_{a a}(t), \\
& \frac{\mathrm{d} N_{\delta}(t)}{\mathrm{d} t}=0, \\
& N_{a t}(0)=2 \sum_{j=1}^{N}\left(1-\mathrm{e}^{-\lambda S_{j}}\right), \quad N_{a a}(0)=\lambda S_{T}-\sum_{j=1}^{N}\left(1-\mathrm{e}^{-\lambda S_{j}}\right), \\
& N_{\delta}(0)=\sum_{j=1}^{N} \mathrm{e}^{-\lambda S_{j}}, \quad N_{r}(0)=0, \quad N_{t t}(0)=0,
\end{aligned}
$$

where $t=0$ (no superscript) is used as shorthand for $t=0^{+}$. The first of these equations integrates to $N_{a t}(t)=N_{a t}(0) /\left(N_{a t}(0) \kappa t+1\right)$. To integrate the second, introduce the combination $U(t)=2 N_{a a}(t)+N_{a t}(t) ; U(t)$ is interpreted as the total number of reactive DSB free ends at time $t$. Combining the first equation with twice the second and integrating gives $U(t)=U(0) /(U(0) \kappa t+1)$ and thus

$$
N_{a a}(t)=\frac{1}{2} U(t)-\frac{1}{2} N_{a t}(t)=\frac{\frac{1}{2} U(0)}{U(0) \kappa t+1}-\frac{\frac{1}{2} N_{a t}(0)}{N_{a t}(0) \kappa t+1} .
$$

The bitelomeric fragment pool size is solved as 


$$
\begin{aligned}
N_{t t}(t) & =\frac{\kappa}{2} \int_{0}^{t} N_{a t}^{2}(\tau) \mathrm{d} \tau=\frac{\kappa}{2} \int_{0}^{t} \frac{N_{a t}^{2}(0)}{\left(N_{a t} \kappa \tau+1\right)^{2}} \mathrm{~d} \tau \\
& =\frac{N_{a t}(0)}{2}\left(1-\frac{1}{N_{a t}(0) \kappa t+1}\right)=\frac{N_{a t}(0)}{2} \frac{N_{a t}(0) \kappa t}{\left(N_{a t}(0) \kappa t+1\right)}, \\
N_{t t}(\infty) & =\frac{N_{a t}(0)}{2} .
\end{aligned}
$$

Eq. (20) is intuitively clear: since edges continue to grab internals until they grab another edge, the final number of bitelomeric fragments must equal half the initial number of edges. Note that $N_{\delta}(\infty)=N_{\delta}\left(0^{+}\right)$and $N_{\delta}(\infty)+$ $N_{t t}(\infty)=N_{\delta}\left(0^{-}\right)$.

The ring pool size $N_{r}(t)$ is obtained from Eq. (14) by integrating $N_{a a}(t)$ in Eq. (18):

$$
\begin{aligned}
N_{r}(t) & =\kappa \int_{0}^{t} N_{a a}(\tau) \mathrm{d} \tau=\kappa \frac{1}{2 \kappa} \ln \left(\frac{U(0) \kappa t+1}{N_{a t}(0) \kappa t+1}\right) \\
& =\frac{1}{2} \ln \left(\frac{U(0) \kappa t+1}{N_{a t}(0) \kappa t+1}\right), \\
N_{r}(\infty) & =\frac{1}{2} \ln \left(\frac{U(0)}{N_{a t}(0)}\right)=\frac{1}{2} \ln \left(1+\frac{2 N_{a a}(0)}{N_{a t}(0)}\right) .
\end{aligned}
$$

The qualitative form of Eq. (22) is reasonable: $N_{r}(\infty)$ increases as $N_{a a}(0)$ increases; it decreases with increasing $N_{a t}(0)$ because edge fragments are expected to pull internals away from ring formation. Cells with many chromosomes are therefore expected to allow the formation of relatively few rings since such cells, when irradiated, contain many edges.

The initial pool sizes shown in Eqs. (16) and (17) have a simple interpretation if one notes that $\lambda S_{T}$ is the expected number of reactive DSBs per cell and $\sum_{j=1}^{N}\left(1-\mathrm{e}^{-\lambda S_{j}}\right)$ is the expected number of chromosomes hit at least once. Thus Eq. (16) states that there are two edges for every chromosome hit and that the number of internals equals the total number of reactive DSBs minus those DSBs spent making edges out of chromosomes (an internal cannot be made from a chromosome until an edge is made first). In Eq. (17), $N_{\delta}(0)$ is the expected number of unhit chromosomes; misrejoined fragment pools are initially empty.

\section{The Fourier transformed SSEB model}

The time-integrals of the basic SSEB equations can be obtained using Fourier transforms. The Fourier transform [15] of the SSEB model is ${ }^{3}$

\footnotetext{
${ }^{3}$ The Fourier transform of $n_{c}(x, t)$ is defined as $\tilde{n}_{c}(k, t)=\int_{0}^{\infty} n_{c}(x, t) \mathrm{e}^{-\mathrm{i} k x} \mathrm{~d} x$, where $\mathrm{i}=\sqrt{-1}$.
} 


$$
\begin{aligned}
& \frac{\partial \tilde{n}_{a t}(k, t)}{\partial t}=-\kappa \tilde{n}_{a t}(k, t) U(t)+2 \kappa \tilde{n}_{a t}(k, t) \tilde{n}_{a a}(k, t), \\
& \frac{\partial \tilde{n}_{a a}(k, t)}{\partial t}=-2 \kappa \tilde{n}_{a a}(k, t) U(t)+2 \kappa \tilde{n}_{a a}^{2}(k, t), \\
& \frac{\partial \tilde{n}_{t t}(k, t)}{\partial t}=\frac{\kappa}{2} \tilde{n}_{a t}^{2}(k, t), \\
& \frac{\partial \tilde{n}_{r}(k, t)}{\partial t}=\kappa \tilde{n}_{a a}(k, t) .
\end{aligned}
$$

Since Eq. (24) is uncoupled from the others we solve it first. Let $\tilde{v}(k, t)=1 / \tilde{n}_{a a}(k, t)$, and note that $\kappa \int_{0}^{t} U(\tau) \mathrm{d} \tau=\ln (U(0) \kappa t+1)$, the second equation then becomes

$$
\begin{aligned}
& -\frac{\dot{\tilde{v}}(k, t)}{\tilde{v}^{2}(k, t)}=-\frac{2 \kappa U(t)}{\tilde{v}(k, t)}+\frac{2 \kappa}{\tilde{v}^{2}(k, t)}, \\
& \dot{\tilde{v}}(k, t)=2 \kappa U(t) \tilde{v}(k, t)-2 \kappa, \\
& \frac{\partial}{\partial t}\left[\tilde{v}(k, t) \exp \left[-2 \kappa \int_{0}^{t} U(\tau) \mathrm{d} \tau\right]=-2 \kappa \exp \left[-2 \kappa \int_{0}^{t} U(\tau) \mathrm{d} \tau\right],\right. \\
& \frac{\partial}{\partial t}\left[\tilde{v}(k, t)(U(0) \kappa t+1)^{-2}\right]=-2 \kappa(U(0) \kappa t+1)^{-2}, \\
& \tilde{v}(k, t)(U(0) \kappa t+1)^{-2}-\tilde{v}(k, 0)=\frac{2}{U(0)}\left[(U(0) \kappa t+1)^{-1}-1\right], \\
& \tilde{v}(k, t)=(U(0) \kappa t+1)(\tilde{v}(k, 0)(U(0) \kappa t+1)-2 \kappa t), \\
& \tilde{n}_{a a}(k, t)=\frac{\tilde{n}_{a a}(k, 0) /(U(0) \kappa t+1)}{U(0) \kappa t+1-\tilde{n}_{a a}(k, 0) 2 \kappa t} \\
& =\frac{\tilde{n}_{a a}(k, 0)}{[U(0) \kappa t+1]\left[\left(U(0)-2 \tilde{n}_{a a}(k, 0)\right) \kappa t+1\right]} \\
& =\frac{\frac{1}{2} U(0)}{U(0) \kappa t+1}-\frac{\frac{1}{2}\left(U(0)-2 \tilde{n}_{a a}(k, 0)\right)}{\left[U(0)-2 \tilde{n}_{a a}(k, 0)\right] \kappa t+1} .
\end{aligned}
$$

This expression for the internal fragment density can be integrated to form the ring density

$$
\begin{aligned}
& \tilde{n}_{r}(k, t)=\kappa \int_{0}^{t} \tilde{n}_{a a}(k, \tau) \mathrm{d} \tau=\frac{1}{2} \ln \left(\frac{U(0) \kappa t+1}{\left[U(0)-2 \tilde{n}_{a a}(k, 0)\right] \kappa t+1}\right), \\
& \tilde{n}_{r}(k, \infty)=\frac{1}{2} \ln \left(\frac{U(0)}{U(0)-2 \tilde{n}_{a a}(k, 0)}\right) .
\end{aligned}
$$


The edge density is given by

$$
\begin{aligned}
& \frac{\partial \tilde{n}_{a t}(k, t)}{\partial t}=\left[2 \kappa \tilde{n}_{a a}(k, t)-\kappa U(t)\right] \tilde{n}_{a t}(k, t), \\
& \frac{\partial \tilde{n}_{a t}(k, t)}{\partial t}-\left[2 \kappa \tilde{n}_{a a}(k, t)-\kappa U(t)\right] \tilde{n}_{a t}(k, t)=0, \\
& \frac{\partial}{\partial t}\left(\tilde{n}_{a t}(k, t) \exp \left[-\int_{0}^{t}\left[2 \kappa \tilde{n}_{a a}(k, \tau)-\kappa U(\tau)\right] \mathrm{d} \tau\right]\right)=0, \\
& \tilde{n}_{a t}(k, t) \exp \left[-\int_{0}^{t}\left[2 \kappa \tilde{n}_{a a}(k, \tau)-\kappa U(\tau)\right] \mathrm{d} \tau\right]=\tilde{n}_{a t}(k, 0), \\
& \tilde{n}_{a t}(k, t)=\tilde{n}_{a t}(k, 0) \exp \left[\int_{0}^{t}\left[2 \kappa \tilde{n}_{a a}(k, \tau)-\kappa U(\tau)\right] \mathrm{d} \tau\right] \\
& =\frac{\tilde{n}_{a t}(k, 0)}{U(0) \kappa t+1} \exp \left[\int_{0}^{t} 2 \kappa \tilde{n}_{a a}(k, \tau) \mathrm{d} \tau\right] \\
& =\frac{\tilde{n}_{a t}(k, 0)}{U(0) \kappa t+1} \frac{\tilde{n}_{a t}(k, 0)}{\left[U(0)-2 \tilde{n}_{a t}(k, 0)\right] \kappa t+1} \\
& =\frac{\left.\tilde{n}_{a a}(k, 0)\right] \kappa t+1}{[U(0)-\tilde{n}(0)} .
\end{aligned}
$$

This solution can be squared and integrated over time to obtain the bitelomeric density, i.e.

$$
\begin{aligned}
\frac{\partial \tilde{n}_{t t}(k, t)}{\partial t} & =\frac{\kappa}{2} \frac{\tilde{n}_{a t}^{2}(k, 0)}{\left[\left(U(0)-2 \tilde{n}_{a a}(k, 0)\right) \kappa t+1\right]^{2}}, \\
\tilde{n}_{t t}(k, t) & =\frac{1}{2} \frac{\tilde{n}_{a t}^{2}(k, 0)}{U(0)-2 \tilde{n}_{a a}(k, 0)}\left(1-\frac{1}{\left(U(0)-2 \tilde{n}_{a a}(k, 0)\right) \kappa t+1}\right) \\
& =\frac{1}{2} \frac{\tilde{n}_{a t}^{2}(k, 0)}{U(0)-2 \tilde{n}_{a a}(k, 0)}\left(\frac{\left(U(0)-2 \tilde{n}_{a a}(k, 0)\right) \kappa t}{\left(U(0)-2 \tilde{n}_{a a}(k, 0)\right) \kappa t+1}\right) \\
& =\frac{1}{2} \frac{\tilde{n}_{a t}^{2}(k, 0) \kappa t}{\left(U(0)-2 \tilde{n}_{a a}(k, 0)\right) \kappa t+1}, \\
\tilde{n}_{t t}(k, \infty) & =\frac{1}{2} \frac{\tilde{n}_{a t}^{2}(k, 0)}{U(0)-2 \tilde{n}_{a a}(k, 0)} .
\end{aligned}
$$

Note that, in contrast to the $n(x, t)$ dependence on $n\left(x^{\prime}, 0\right)$ for all $x^{\prime} \leqslant x$, the $\tilde{n}(k, t)$ 's depend on the $\tilde{n}(k, 0)$ 's only for the same value of $k$, i.e. sinusoidal functions are here playing the same decoupling role normal modes play in 
linear problems. Also note that when $k=0, N_{c}(t)=\tilde{n}_{c}(0, t)$ and Eqs. (33)-(39) reduce to the pool size solutions.

The preceeding equations express the Fourier transformed densities in terms of the initial densities. Assuming low LET, the EtBr model specifies the initial fragment densities. The Fourier transform of the initial edge density is

$$
\begin{aligned}
\tilde{n}_{a t}(k, 0) & =\int_{0}^{\infty} \sum_{j=1}^{N} 2 \mathrm{e}^{-i k x} u_{0}\left(x, S_{j}\right) \lambda \mathrm{e}^{-\lambda x} \mathrm{~d} x \\
& =2 \sum_{j=1}^{N} \int_{0}^{S_{j}} \mathrm{e}^{-(i k+\lambda) x} \lambda \mathrm{d} x=\frac{2 \lambda}{\lambda+i k} \sum_{j=1}^{N}\left(1-\mathrm{e}^{-(i k+\lambda) S_{j}}\right) .
\end{aligned}
$$

If the dose is large enough that each chromosome is hit at least once (the first high-dose condition), the initial edge sizes become exponentially distributed since

$$
\sum_{j=1}^{N}\left(1-\mathrm{e}^{-(i k+\lambda) S_{j}}\right) \approx N \Rightarrow \tilde{n}_{a t}(k, 0) \approx 2 N \frac{\lambda}{\lambda+i k} \Rightarrow n_{a t}(x, 0) \approx 2 N \lambda \mathrm{e}^{-\lambda x} .
$$

The first high-dose condition implies that there are $2 N$ edge fragments and $\lambda S_{T}-N$ internal fragments at $t=0$. The Fourier transform of the initial internal fragment density is

$$
\begin{aligned}
\tilde{n}_{a a}(k, 0) & =\int_{0}^{\infty} \sum_{j=1}^{N} \lambda\left(S_{j}-x\right) \mathrm{e}^{-i k x} u_{0}\left(x, S_{j}\right) \lambda \mathrm{e}^{-\lambda x} \mathrm{~d} x \\
& =\lambda^{2} \sum_{j=1}^{N} \int_{0}^{S_{j}}\left(S_{j}-x\right) \mathrm{e}^{-(i k+\lambda) x} \mathrm{~d} x \\
& =\lambda^{2} \sum_{j=1}^{N} \frac{S_{j}}{\lambda+i k}-\frac{1}{\lambda+i k} \int_{0}^{S_{j}} \mathrm{e}^{-(i k+\lambda) x} \mathrm{~d} x \\
& =\lambda^{2} \sum_{j=1}^{N} \frac{S_{j}}{\lambda+i k}-\frac{1}{(\lambda+i k)^{2}}\left(1-\mathrm{e}^{-(i k+\lambda) S_{j}}\right) \\
& =\lambda^{2} \frac{S_{T}}{\lambda+i k}-\lambda^{2}\left(\frac{N-\sum_{j=1}^{N} \mathrm{e}^{-(i k+\lambda) S_{j}}}{(\lambda+i k)^{2}}\right) .
\end{aligned}
$$

Assuming the first high-dose condition,

$$
\tilde{n}_{a a}(k, 0)=\frac{\lambda^{2} S_{T}}{\lambda+i k}-\frac{N \lambda^{2}}{(\lambda+i k)^{2}} .
$$


For internals to be exponentially distributed, the second high-dose condition, $\lambda S_{T}-N \approx \lambda S_{T}$, is also needed so that

$$
\begin{aligned}
\tilde{n}_{a a}(k, 0) & =\lambda^{2}\left(\frac{S_{T}}{\lambda+i k}-\frac{N}{(\lambda+i k)^{2}}\right)=\lambda^{2}\left(\frac{S_{T}(\lambda+i k)-N}{(\lambda+i k)^{2}}\right) \\
& \approx \lambda^{2}\left(\frac{S_{T}(\lambda+i k)}{(\lambda+i k)^{2}}\right)=\lambda S_{T} \frac{\lambda}{(\lambda+i k)} .
\end{aligned}
$$

The second high-dose condition implies that $N_{a a}(0)=\lambda S_{T}$ while the first highdose condition implies that $N_{a a}(0)=\lambda S_{T}-N$. When both conditions hold, the second high-dose condition implies that these differences in $N_{a a}(0)$ are negligible. Furthermore, since the second high-dose condition implies $\lambda \gg N / S_{T}$, we can add $N / S_{T}$ to $\lambda$ and redefine it as the number of 'genome interruptions' (reactive DSBs plus pairs of natural telomeres) per base pair.

Eqs. (33), (34), (36) and (38), together with the initial conditions (40) and (42) form, in principle, a full solution of the SSEB equations. Numeric evaluation of these solutions will be illustrated in Section 7. Section 6 gives analytic solutions for the high-dose limit in which the initial conditions have the simpler form (41) and (44).

\section{Exponential initial conditions}

In applications, e.g. to PFGE measurements, numerical integration of Eqs. (4)-(10) or numerical evaluation of Eqs. (33), (34), (36), (38), (40) and (42) is needed. A useful check on numerical methods is the exact analytic solution in the high-dose case, which we now obtain using inverse Fourier transforms.

The pool size solutions remain as given in Section 4, namely

$$
\begin{aligned}
& N_{a t}(t)=\frac{N_{a t}(0)}{N_{a t}(0) \kappa t+1}, \\
& N_{a a}(t)=\frac{\frac{1}{2} U(0)}{U(0) \kappa t+1}-\frac{\frac{1}{2} N_{a t}(0)}{N_{a t}(0) \kappa t+1}=\frac{N_{a a}(0)}{\left[N_{a t}(0) \kappa t+1\right][U(0) \kappa t+1]}, \\
& N_{t t}(t)=\frac{N_{a t}(0)}{2}\left(1-\frac{1}{N_{a t}(0) \kappa t+1}\right)=\frac{N_{a t}(0)}{2} N_{a t}(t) \kappa t, \\
& N_{r}(t)=\frac{1}{2} \ln \left(\frac{U(0) \kappa t+1}{N_{a t}(0) \kappa t+1}\right) .
\end{aligned}
$$

Assuming exponentially distributed initial fragment size distributions (first and second high-dose conditions), the initial conditions $\tilde{n}_{a t}(k, 0)=N_{a t}(0) \lambda /(i k+\lambda)$ and $\tilde{n}_{a a}(k, 0)=N_{a a}(0) \lambda /(i k+\lambda)$ yield 


$$
\begin{aligned}
\tilde{n}_{a t}(k, t) & =\frac{\tilde{n}_{a t}(k, 0)}{\left[U(0)-2 \tilde{n}_{a a}(k, 0)\right] \kappa t+1} \\
& =\frac{N_{a t}(0) \lambda /(i k+\lambda)}{\left[2 N_{a a}(0) i k /(i k+\lambda)+N_{a t}(0)\right] \kappa t+1} \\
& =\frac{N_{a t}(0) \lambda}{(U(0) \kappa t+1) i k+\lambda\left(1+N_{a t}(0) \kappa t\right)}=\frac{N_{a t}(t) \lambda(t)}{i k+\lambda(t)}, \\
n_{a t}(x, t) & =N_{a t}(t) \lambda(t) \mathrm{e}^{-\lambda(t) x},
\end{aligned}
$$

where

$$
\lambda(t)=\lambda \frac{N_{a t}(0) \kappa t+1}{U(0) \kappa t+1} \quad \text { and } \quad \lambda(\infty)=\lambda \frac{N_{a t}(0)}{U(0)}=\lambda \frac{2 N}{2 \lambda S_{T}}=\frac{N}{S_{T}} .
$$

The edge density is thus exponentially distributed with a shape parameter $\lambda(t)$ that decays hyperbolically (i.e. as $A /(B t+C)$ ) at early times but then converges at $t=\infty$ to $N / S_{T}$, the "natural genome interruption frequency" of the organism, i.e. the inverse of the average chromosome length. Thus, at very large times, the average edge fragment length approaches, but does not exceed, the organisms average chromosome length. ${ }^{4}$

For internal fragments,

$$
\begin{aligned}
\tilde{n}_{a a}(k, t)= & \frac{\tilde{n}_{a a}(k, 0)}{[U(0) \kappa t+1]\left[\left(U(0)-2 \tilde{n}_{a a}(k, 0)\right) \kappa t+1\right]} \\
= & \frac{N_{a a}(0) \lambda /(i k+\lambda)}{[U(0) \kappa t+1]\left[\left(2 N_{a a}(0) i k /(i k+\lambda)+N_{a t}(0)\right) \kappa t+1\right]} \\
= & \frac{N_{a a}(0) \lambda}{[U(0) \kappa t+1]\left[(U(0) \kappa t+1) i k+\lambda\left(1+N_{a t}(0) \kappa t\right)\right]} \\
= & \frac{N_{a a}(0)}{[U(0) \kappa t+1]\left[1+N_{a t}(0) \kappa t\right]} \\
& \times\left(\frac{\lambda\left[1+N_{a t}(0) \kappa t\right]}{\left[(U(0) \kappa t+1) i k+\lambda\left(1+N_{a t}(0) \kappa t\right)\right]}\right) \\
= & N_{a a}(t) \frac{\lambda\left[1+N_{a t}(0) \kappa t\right]}{\left[(U(0) \kappa t+1) i k+\lambda\left(1+N_{a t}(0) \kappa t\right)\right]} \\
= & N_{a a}(t) \frac{\lambda(t)}{i k+\lambda(t)} .
\end{aligned}
$$

The inverse transform, by inspection, is therefore

$$
n_{a a}(x, t)=N_{a a}(t) \lambda(t) \mathrm{e}^{-\lambda(t) x},
$$

i.e. the internal fragment density also remains exponential with a time-dependent shape parameter $\lambda(t)$. Internals and edges have the same $\lambda(t)$ dependence. Why is this? Note that when internals react with edges or internals they act to

\footnotetext{
${ }^{4}$ Recall that the mean of an exponential $p(x, t)=\lambda(t) \mathrm{e}^{-\lambda(t) x}$ is $1 / \lambda(t)$.
} 
drive the densities toward larger $x$ values and thus smaller $\lambda(t)$ values. Although internals drive themselves toward large $x$ twice as fast as they drive edges, internals are also annihilated by edges twice as fast as edges annihilate themselves. These effects appear to cancel so that edges and internals have the same $\lambda(t)$.

Since the number of reactive DSBs per base pair,

$$
\lambda_{a}(t)=\frac{U(t) / 2}{S_{T}}=\frac{1}{S_{T}} \frac{U(0) / 2}{U(0) \kappa t+1},
$$

is hyperbolic, the bilinear density shape parameter $\lambda(t)$ in Eq. (51) is not equivalent to $\lambda_{a}(t)$. From its form as $t \rightarrow \infty$, it appears that $\lambda(t)$ represents the number of genome interruptions per base pair, regardless of whether the interruption is a reactive DSB or two natural telomeres. This interpretation of $\lambda(t)$ is consistent with our definition of $\lambda$ as the initial number of reactive DSBs per base pair since the second high-dose condition implies that the initial number of genome interruptions approximately equals the initial number of reactive DSBs. However, at large times, when the majority of reactive DSBs have rejoined, the difference between $\lambda(t)$ and $\lambda_{a}(t)$ is significant.

Assuming edges and internals are initially exponential, the ring solution (34) becomes

$$
\begin{aligned}
\tilde{n}_{r}(k, t) & =\frac{1}{2} \ln \left(\frac{U(0) \kappa t+1}{\left[U(0)-2 \tilde{n}_{a a}(k, 0)\right] \kappa t+1}\right) \\
& =\frac{1}{2} \ln \left(\frac{U(0) \kappa t+1}{\left[2 N_{a a}(0) i k /(i k+\lambda)+N_{a t}(0)\right] \kappa t+1}\right) \\
& =\frac{1}{2} \ln \left(\frac{(U(0) \kappa t+1)(i k+\lambda)}{\left[2 N_{a a}(0) i k+(i k+\lambda) N_{a t}(0)\right] \kappa t+(i k+\lambda)}\right) \\
& =\frac{1}{2} \ln \left(\frac{(U(0) \kappa t+1)(i k+\lambda)}{(U(0) \kappa t+1) i k+\lambda\left(1+N_{a t}(0) \kappa t\right)}\right) \\
& =\frac{1}{2} \ln \left(\frac{i k+\lambda}{i k+\lambda(t)}\right), \\
n_{r}(x, t) & =\frac{1}{2 x}\left(\mathrm{e}^{-\lambda(t) x}-\mathrm{e}^{-\lambda x}\right), \\
n_{r}(x, \infty) & =\frac{1}{2 x}\left(\mathrm{e}^{-\left(N / S_{T}\right) x}-\mathrm{e}^{-\lambda x}\right)
\end{aligned}
$$

and, by similar arguments for bitelomeres, Eq. (38) becomes

$$
\begin{aligned}
\tilde{n}_{t t}(k, t) & =N_{t t}(t)\left(\frac{\lambda}{\lambda+i k}\right)\left(\frac{\lambda(t)}{i k+\lambda(t)}\right) \\
& =N_{t t}(t) \frac{\lambda \lambda(t)}{\lambda-\lambda(t)}\left(\frac{1}{i k+\lambda(t)}-\frac{1}{i k+\lambda}\right),
\end{aligned}
$$




$$
\begin{aligned}
n_{t t}(x, t) & =N_{t t}(t) \frac{\lambda \lambda(t)}{\lambda-\lambda(t)}\left(\mathrm{e}^{-\lambda(t) x}-\mathrm{e}^{-\lambda x}\right), \\
n_{t t}(x, \infty) & =\frac{N_{a t}(0)}{2} \frac{\lambda\left(N / S_{T}\right)}{\lambda-\left(N / S_{T}\right)}\left(\mathrm{e}^{-\left(N / S_{T}\right) x}-\mathrm{e}^{-\lambda x}\right) .
\end{aligned}
$$

Focusing on just the final solutions $(t=\infty)$, note that in the limit of very high doses $(\lambda=\infty)$ the misrejoined fragment number densities approach

$$
\begin{aligned}
& n_{t t}(x, \infty)=\frac{N^{2}}{S_{T}} \mathrm{e}^{-\left(N / S_{T}\right) x}, \\
& n_{r}(x, \infty)=\frac{1}{2 x} \mathrm{e}^{-\left(N / S_{T}\right) x},
\end{aligned}
$$

as illustrated in Fig. 2. If we integrate the corresponding fragment mass densities

$$
\begin{aligned}
& m_{t t}(x, \infty)=x n_{t t}(x, \infty)=x \frac{N^{2}}{S_{T}} \mathrm{e}^{-\left(N / S_{T}\right) x}, \\
& m_{r}(x, \infty)=x n_{r}(x, \infty)=\frac{1}{2} \mathrm{e}^{-\left(N / S_{T}\right) x},
\end{aligned}
$$

from $x=0$ to $\infty$ we obtain $M_{t t}(\infty)=S_{T}$ and $M_{r}(\infty)=S_{T} /(2 N)$, i.e. in the limit of high-doses, the total ring mass approaches one-half the average chromosome mass. Note that mass is not conserved $\left(S_{T}+\left(S_{T} / 2 N\right)>S_{T}\right)$ because, in forming the SSEB model, the ring term was dropped as an internal fragment degradation flux (source term), but kept as a ring synthesis flux (target term), see Eqs. (5) and (7). If we include the term in both locations, the high-dose limit $M_{t t}(\infty) \approx S_{T}-\left(S_{T} / 2 N\right)$ should result. The total ring and bitelomere masses should not be confused with $M(t)$, the expected number of misrejoinings per cell.

\section{Numerical results}

We recently developed the Sax-Markov binary eurejoining/misrejoining (SMBE) model in order to explain high dose (80-160 Gy) PFGE data as well as moderate dose (1-5 Gy) chromosome aberration data [12]. The SMBE model predicts a quadratic-linear $M(\infty)$ dose-response in the range of 1-160 Gy. ${ }^{5}$ In the following sections we shall use the SMBE algorithm ${ }^{6}$ to compute $\lambda$ in the EtBr model as a function of dose, i.e. $\lambda=M(\infty) / S_{T}$ with $M(\infty)$ taken from the SMBE model.

\footnotetext{
${ }^{5}$ Since the SMBE model ignores one-track action, the numerical results that follow will be limited to doses greater than approximately $1 \mathrm{~Gy}$.

${ }^{6}$ The SMBE algorithm in $\mathrm{C}$ is available from radivot@musc.edu.
} 

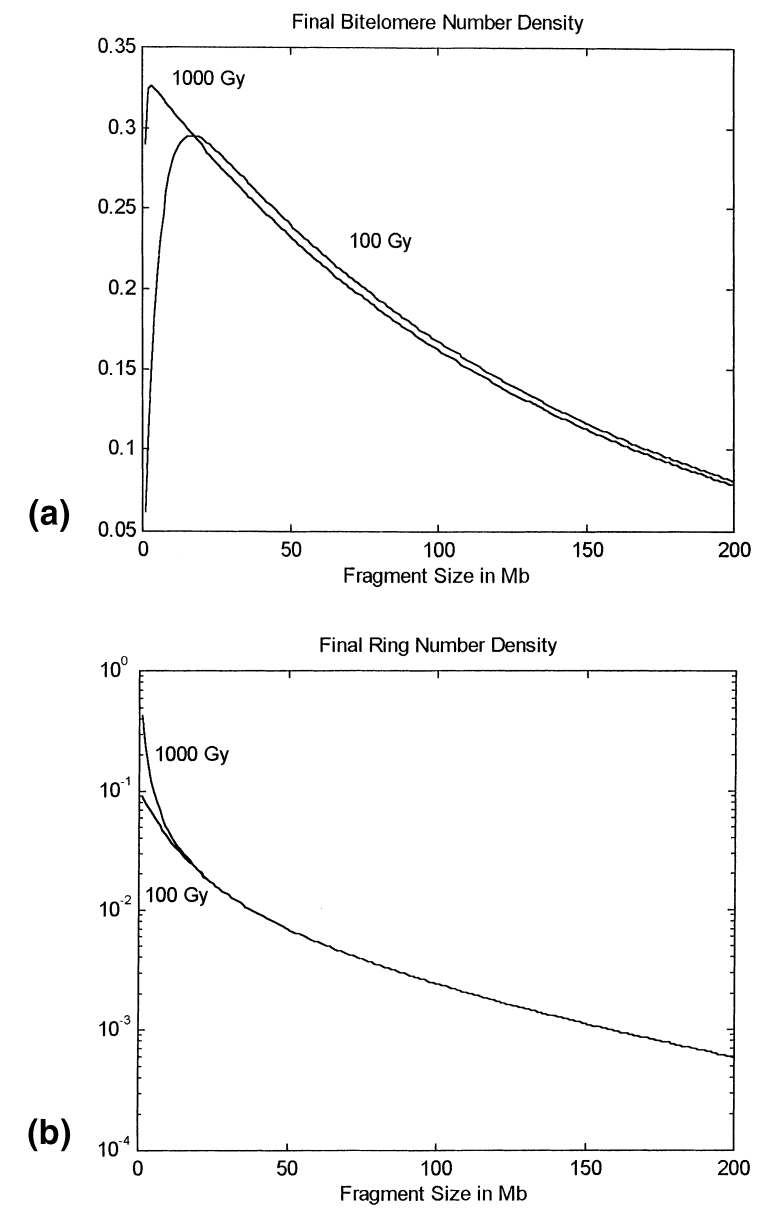

Fig. 2. The final bitelomeric fragment number density $n_{t t}(x, \infty)$ (a) has an exponential high-dose limiting form. The density of small rings continues to increase with very high-doses (b).

\subsection{Ring yields}

To examine ring yields we: (1) formed $\lambda$ using the SMBE model at several doses; (2) used the $\lambda$ 's and the $S_{j}$ 's of male human cells during $G_{0}$ to compute the initial fragment pool sizes (Eq. (16)); and (3) used the initial pool sizes to compute the final misrejoined fragment pool sizes according to Eqs. (20) and (22). The results (Table 1) suggest that for doses less than 5 Gy, Eqs. (16), (20) and (22) are approximately

$$
N_{a t}(0) \approx 2 \lambda S_{T}=2 M(\infty),
$$


Table 1

The initial and final fragment pool sizes in human (male) cells resting in $G_{0}$ were computed as described in the text. The SMBE parameter values used to compute $M(\infty)$, and thus $\lambda=M(\infty) / S_{T}$, were $p=0.33, G=40$ and $\eta=375$, where $p$ is the fraction of DSBs that are active, $G$ is the yield in DSBs per Gy per human cell, $\eta$ is the number of DSB sites within the nucleus and $M(\infty)$ is the expected number of misrejoinings (reactive DSBs) per cell [12]. Note that the first high dose condition (all chromosomes hit at least once) is satisfied at $D=50 \mathrm{~Gy}$ and that the second high dose condition (many times more internals than chromosomes) is satisfied at $D=100 \mathrm{~Gy}$

\begin{tabular}{llllllll}
\hline Dose in Gray & 1 & 2 & 5 & 10 & 20 & 50 & 100 \\
$M(\infty)$ & 0.3 & 1.2 & 7.3 & 27.4 & 96.3 & 427.7 & 1099.2 \\
$\lambda \mathrm{Mb}^{-1}$ & 0.00005 & 0.00019 & 0.0012 & 0.0043 & 0.015 & 0.067 & 0.173 \\
$N_{a t}(0)$ & 0.62 & 2.41 & 13.37 & 39.64 & 75.93 & 91.62 & 92.00 \\
$N_{a a}(0)$ & 0.001 & 0.019 & 0.65 & 7.58 & 58.35 & 381 & 1053 \\
$N_{r}(\infty)$ & 0.002 & 0.008 & 0.046 & 0.162 & 0.465 & 1.117 & 1.587 \\
$N_{t t}(\infty)$ & 0.3 & 1.2 & 6.7 & 19.8 & 38.0 & 45.8 & 46.0 \\
\hline
\end{tabular}

$$
\begin{aligned}
& N_{a a}(0) \approx \sum_{j=1}^{N} \frac{\lambda^{2} S_{j}^{2}}{2} \\
& N_{t t}(\infty)=\frac{N_{a t}(0)}{2} \approx M(\infty), \\
& N_{r}(\infty) \approx \frac{N_{a a}(0)}{N_{a t}(0)} \approx \frac{M(\infty)}{4} \sum_{j=1}^{N}\left(\frac{S_{j}}{S_{T}}\right)^{2} .
\end{aligned}
$$

The ring dose-response is thus predicted to equal the bitelomere dose-response multiplied by a constant $\frac{1}{4} \sum_{j=1}^{N}\left(S_{j} / S_{T}\right)^{2}$ that increases with the dispersion of the chromosome sizes. At one extreme, when one chromosome approaches size $S_{T}$ and all others approach zero, the proportionality converges to $\frac{1}{4}$. At the other extreme, if all chromosomes have the same size, the proportionality becomes $1 / 4 N$. For human chromosomes the latter of these extremes approximates the full expression in (67), underestimating the ring yields by only about $10 \%$.

The results shown in Table 1 indicate that rings are almost completely negligible (see also Fig. 2). However, it must be kept in mind that SSEB ignores proximity effects due to chromosome sites (the localization of interphase chromosomes to nuclear subdomains) and therefore will underestimate the true number of rings [16]. ${ }^{7}$ If each chromosome is confined to exist in one of $\eta_{c}$ nuclear subdomains, and if the chromosome size distribution within each subdomain is still well represented by the mean size for the organism, then an extension of our arguments gives the estimate $N_{r}(\infty) \approx \eta_{c} M(\infty) / 4 N$. This estimate is in excellent agreement with an estimate obtained by very different, Monte Carlo methods [17]. The Monte Carlo methods, combined with chro-

\footnotetext{
${ }^{7}$ The SSEB model also ignores the fact that small internals should close faster than large internals. The SMBE model accounts for DSB sites (the localization of DSB free ends to nuclear subcompartments) but not chromosome sites.
} 
mosome aberration data, indicate $\eta_{c}$ is about 13 [17]. Applying this correction factor of 13 in Table 1, and comparing $N_{r}(\infty)$ with $N_{t t}(\infty)$ shows that, even with proximity effects taken into account, the neglect of a ring production source term in Eq. (4) is a reasonable approximation.

\subsection{Flow karyotypes}

Experimentally, flow karyotypes have been used to study DNA fragment sizes after irradiation and misrejoining [10]. For doses of approximately 5 Gy, the final number density can be readily separated into a misrejoined signal and a eurejoined signal. These separate signals have been used in the past to form two separate misrejoining estimates - see the peak reduction algorithm (PRA) and background number algorithm (BNA) developed by Dietzel et al. [10]. We now discuss potential applications of the present model to flow karyotype experiments.

In Section 6 we derived analytic SSEB solutions using the high dose limiting form of the EtBr model - exponential initial conditions valid at doses greater than about $100 \mathrm{~Gy}$ for humans, see Table 1. At lower doses the exact form of the EtBr model must be used. Though it may be possible to solve the SSEB model analytically using the exact form of the EtBr model as the initial condition, a numerical approach is preferable because it is more general, i.e. any initial conditions can be specified. The SSEB solutions can be evaluated numerically as follows:

1. Use the EtBr model to convert $n_{\delta}\left(x, 0^{-}\right)$just before irradiation to $n_{\delta}\left(x, 0^{+}\right)$, $n_{a t}\left(x, 0^{+}\right)$and $n_{a a}\left(x, 0^{+}\right)$just after irradiation. The value of $\lambda$ in the $\mathrm{EtBr}$ model depends on dose according to the SMBE model, i.e. $\lambda=M(\infty) / S_{T}$.

2. Take fast Fourier transforms (FFTs) of $n_{a t}\left(x, 0^{+}\right)$and $n_{a a}\left(x, 0^{+}\right)$to form $\tilde{n}_{a t}\left(k, 0^{+}\right)$and $\tilde{n}_{a a}\left(k, 0^{+}\right){ }^{8}$

3. Carry out pointwise $k$-vector multiplications, additions and divisions according to the formulas provided in Section 5. Recognize that $U(0)=$ $2 M(\infty)$ from the SMBE model.

4. Inverse transform the resulting $k$-vectors to yield the $x$ vector solutions.

We executed this procedure for human chromosomes irradiated with doses of 1, 5, 10, 20 and 50 Gy using $\Delta x=1 \mathrm{Mb}$ and vector lengths of 1024. In Fig. 3 we see that the affect of $1 \mathrm{~Gy}$ is indistinguishable from unirradiated controls. Thus flow karyotypes are not likely to be useful at doses less than 1 or 2 Gy. Figs. 4 and 5 provide plots of the initial and final fragment densities, respectively, for doses of 5, 10, 20 and $50 \mathrm{~Gy}$. Comparing the misrejoined karyotype at $50 \mathrm{~Gy}$ with its high dose limit in Fig. 2(a), we see that the shape of the misrejoined frag-

\footnotetext{
${ }^{8}$ FFTs require evenly spaced samples preferably padded with zeros to a power of two. The high dose limit $n_{t t}(x, \infty)=\left(N^{2}\right) /\left(S_{T}\right) \mathrm{e}^{\left(S_{T}\right) /(N) x}$ suggests that the vector length $2^{k}$ should be chosen such that $2^{k} \Delta x$ lies between 5 and 10 times the average chromosome size $S_{T} / N$.
} 


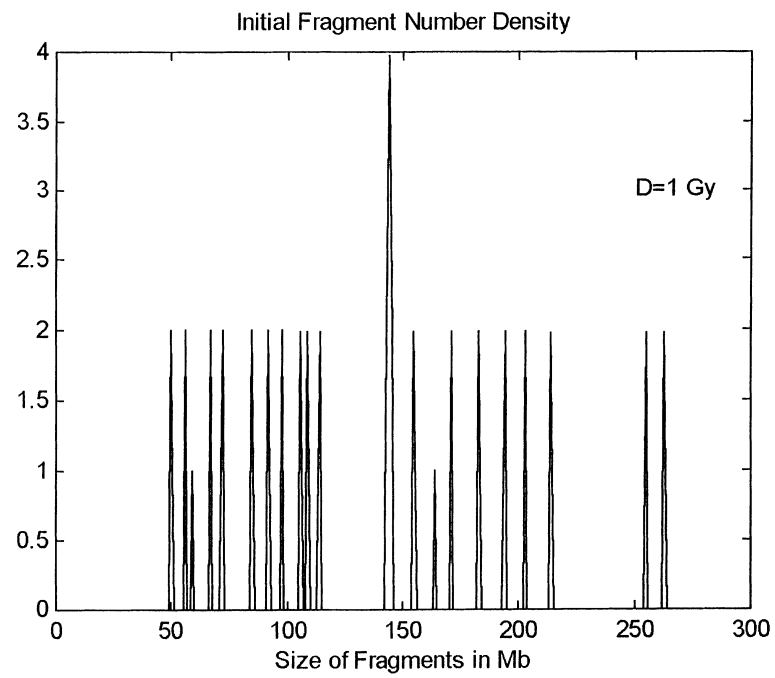

Fig. 3. A dose of $1 \mathrm{~Gy}$ produces an initial fragment size distribution indistinguishable from a dose of 0 Gy. Male human $G_{1}$ cells were used in conjunction with the SMBE model as in Table 1 .

ment signal continues to change with dose, even at very high doses. The SSEB model offers a means of converting this shape information into estimates of the number of misrejoined DSBs per Mb, i.e. SSEB solutions can be fit to high-dose flow karyotypes to arrive at distribution-based misrejoining estimates.

\subsection{PFGE experiments}

In other experiments involving pulsed-field gel electrophoresis (PFGE) to determine fragment sizes, one uses restriction enzymes to cut the DNA of the human genome at specific sites, thus creating DNA fragment sizes small enough to enter pulsed-field gels $[18,19]$. In these experiments, the DNA is digested after the cells are killed, i.e. just before separation on the pulsed-field gel. We shall refer to DNA fragments that result from restriction-enzyme cutting of unirradiated chromosomes as 'cut-somes'. Though cut-somes are much smaller than chromosomes, the size changes cut-somes undergo as a result of irradiation and misrejoining can be modeled with the same formalism as in the case of chromosomes. This rather surprising feature simply reflects the fact that a cut-some also has two 'terminals' (ends) that, like telomeres, are inert during the process of DSB induction and misrejoining. The previous sections thus apply to cut-somes by replacing 'telomere' with 'cut-some terminal' and 'chromosome' with 'cut-some'.

We shall consider experiments using restriction enzymes that have comparatively long recognition sequences for which the average cut-some size is several $\mathrm{Mb}$ [2]. Since the number of cut-somes is typically quite large, a description of the cut-some density in terms of Dirac delta functions is no longer appropri- 

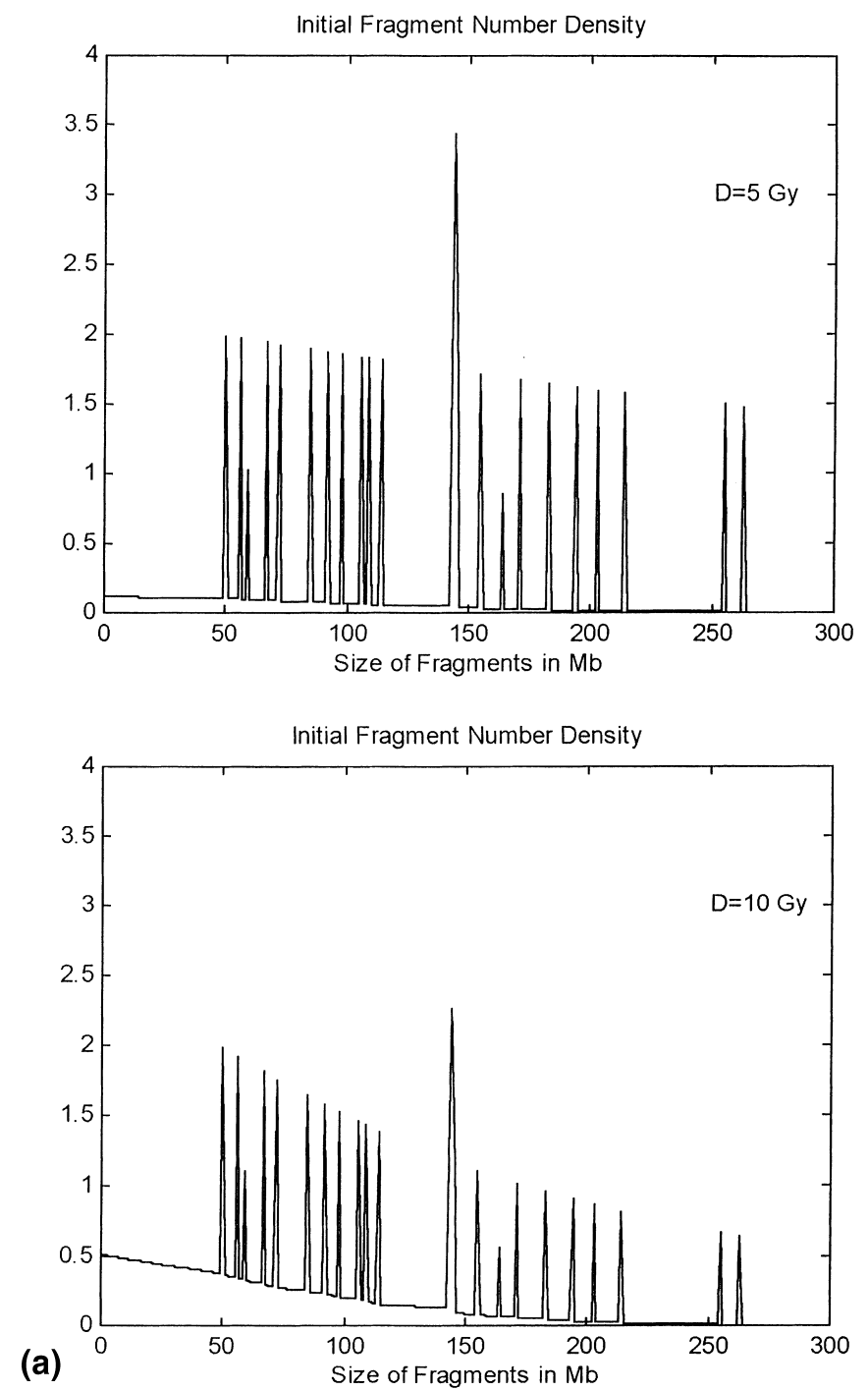

Fig. 4. The initial fragment size distributions at $t=0^{+}$are illustrated here for acute low LET doses of (a) 5,10 , and (b) 20 and $50 \mathrm{~Gy}, \lambda=M(\infty) / S_{T}$ as in Table 1 .

ate. Instead we shall treat cut-some distributions as continuous density functions. To convert the cut-some density at $t=0^{-}$, which we denote by $n_{\delta}\left(s, 0^{-}\right)$, to initial edge, internal and delta densities at $t=0^{+}$(just after acute irradiation), we can use a modified form of the EtBr model in which the sum over $N$ chromosomes is replaced by an integral over the density of cut-somes. The result is that Eqs. (9) and (10) are replaced by 

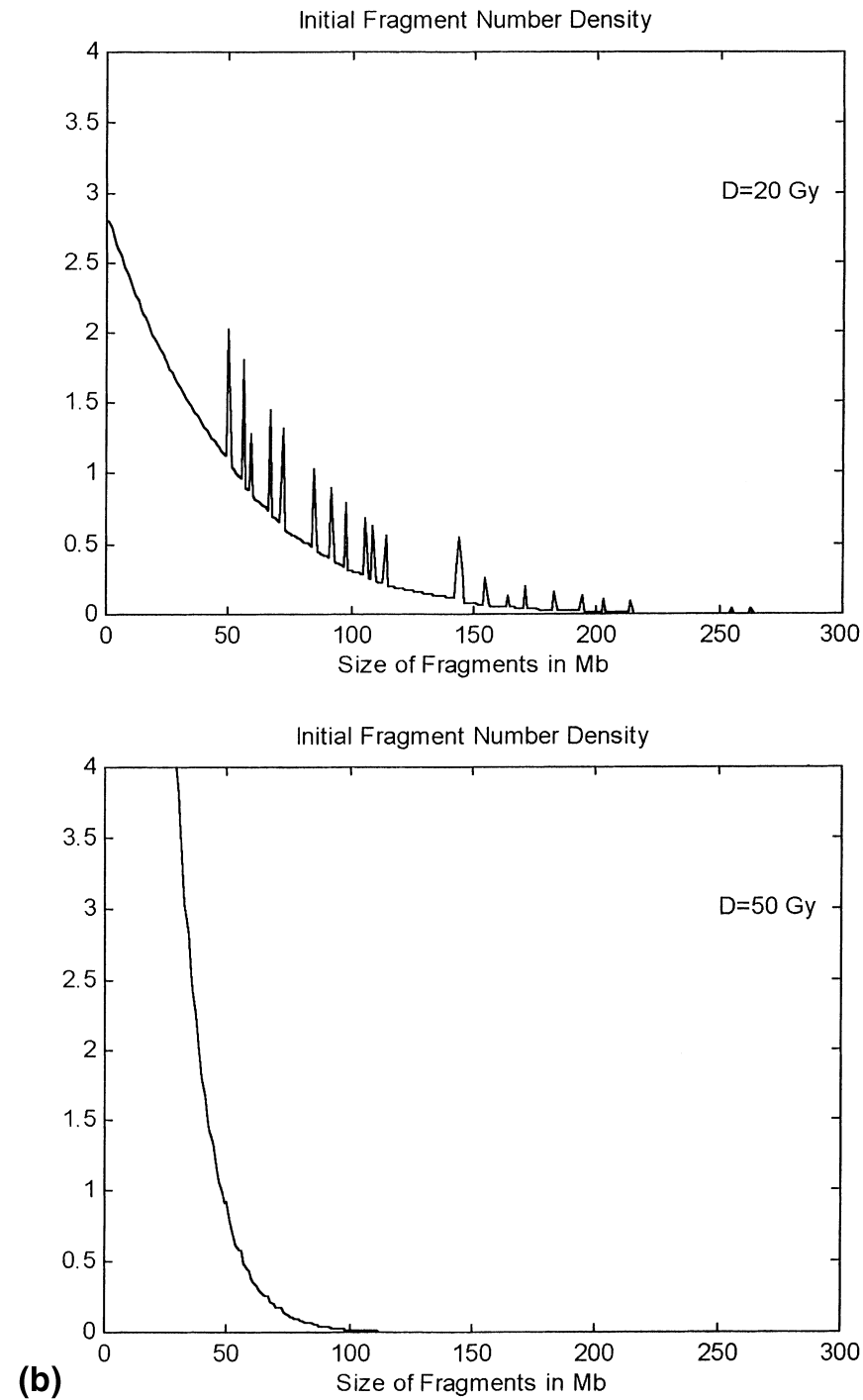

Fig. 4. (continued)

$$
\begin{aligned}
& n_{a t}\left(x, 0^{+}\right)=2 \lambda \mathrm{e}^{-\lambda x} \int_{x}^{\infty} n_{\delta}\left(s, 0^{-}\right) \mathrm{d} s, \\
& n_{a a}\left(x, 0^{+}\right)=\lambda^{2} \mathrm{e}^{-\lambda x} \int_{x}^{\infty} s n_{\delta}\left(s, 0^{-}\right) \mathrm{d} s-\lambda^{2} \mathrm{e}^{-\lambda x} x \int_{x}^{\infty} n_{\delta}\left(s, 0^{-}\right) \mathrm{d} s,
\end{aligned}
$$



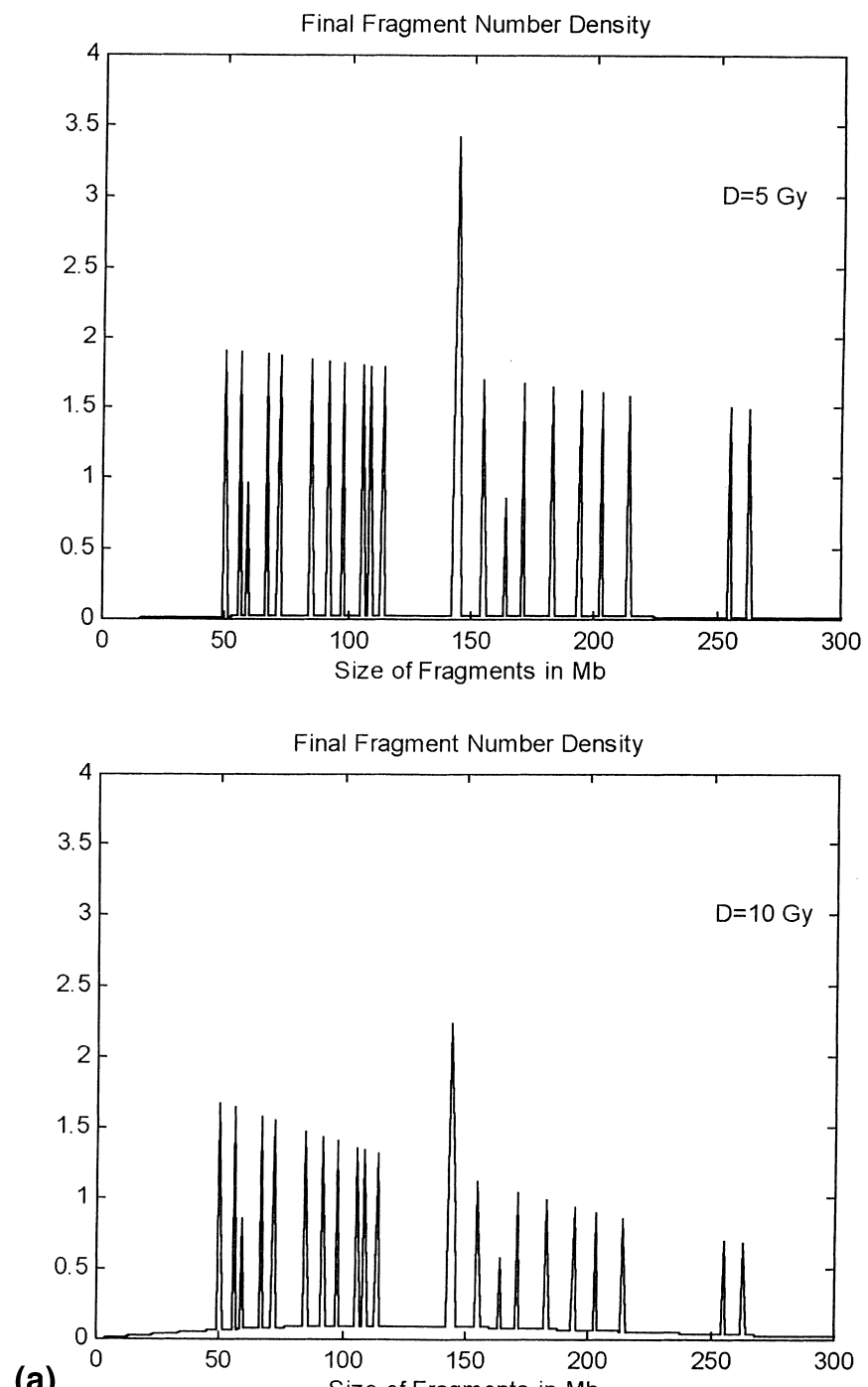

Fig. 5. The final SSEB fragment size densities were evaluated numerically using the initial fragment size densities of Fig. 4. The 'background' density between the peaks is the final misrejoined fragment density, i.e. the sum of the final ring and bitelomere densities. The maximum of this final misrejoined density is seen to move toward smaller $x$ as dose increases between 20 and $50 \mathrm{~Gy}$, consistent with Fig. 2.

$$
n_{\delta}\left(x, 0^{+}\right)=\mathrm{e}^{-\lambda x} n_{\delta}\left(x, 0^{-}\right)
$$

where $\lambda=M(\infty) / S_{T}$ is again defined as a function of dose by the SMBE model. The integrals in Eqs. (68)-(70) can also be expressed as 

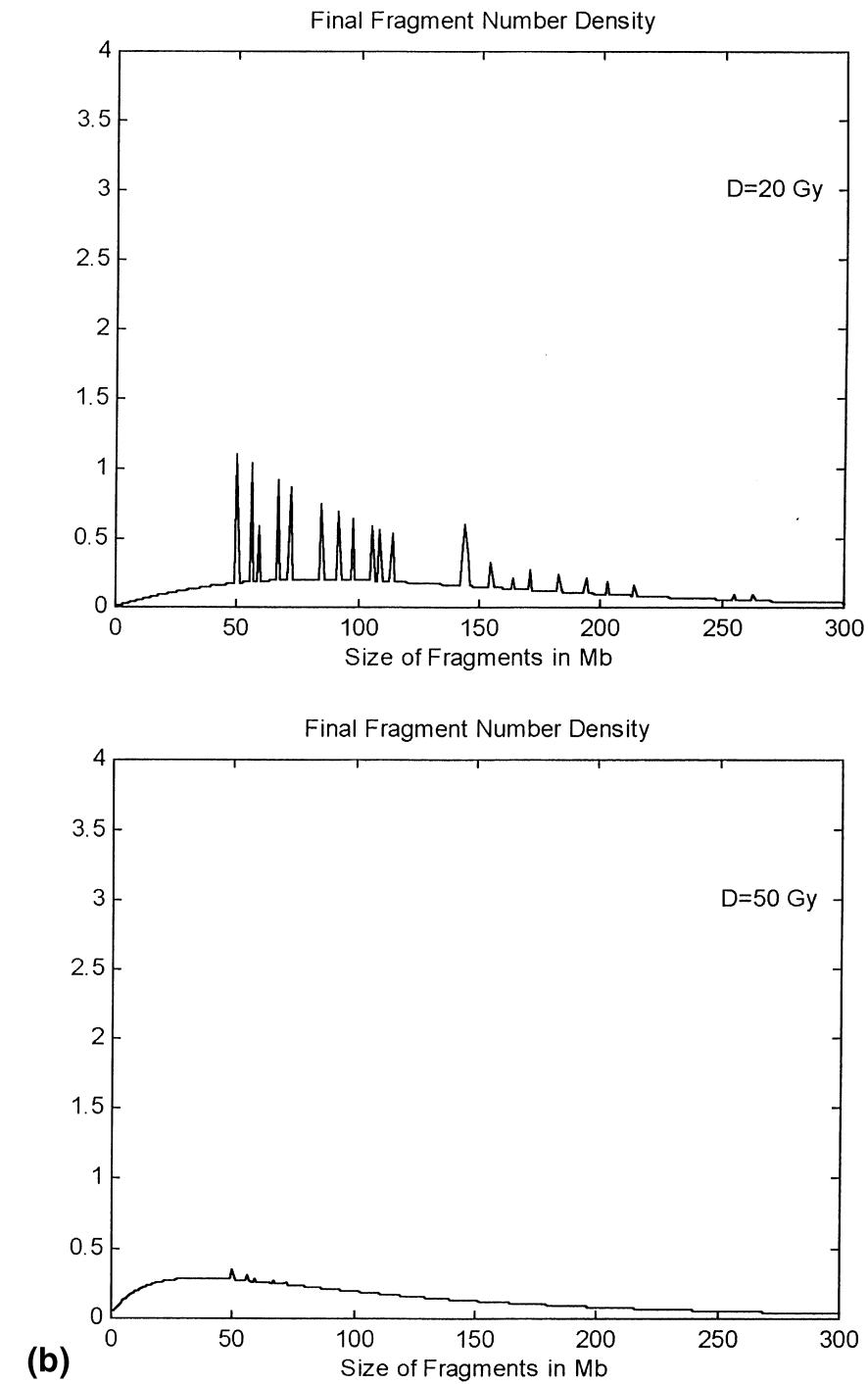

Fig. 5. (continued)

$$
\begin{aligned}
& \int_{x}^{\infty} s n_{\delta}\left(s, 0^{-}\right) \mathrm{d} s=S_{T}-\int_{0}^{x} s n_{\delta}\left(s, 0^{-}\right) \mathrm{d} s, \\
& \int_{x}^{\infty} n_{\delta}\left(s, 0^{-}\right) \mathrm{d} s=N-\int_{0}^{x} n_{\delta}\left(s, 0^{-}\right) \mathrm{d} s,
\end{aligned}
$$


where $N$ is the expected number of cut-somes per cell. These forms are useful in numerical evaluations. We next show that misrejoining estimates cannot be obtained from cut-some size distributions that are exponential (as would occur if the cut-somes are small compared to chromosomes and the recognition sequences are randomly located in the genome). We begin by substituting the form $n_{\delta}\left(s, 0^{-}\right)=\lambda_{0} S_{T} \lambda_{0} \mathrm{e}^{-\lambda_{0} s}$ into Eqs. (68)-(70) to obtain the initial state of the SSEB model

$$
\begin{aligned}
& n_{\delta}\left(x, 0^{+}\right)=\lambda_{0}^{2} S_{T} \mathrm{e}^{-\left(\lambda_{0}+\lambda\right) x}, \\
& n_{a t}\left(x, 0^{+}\right)=2 \lambda_{0} S_{T} \lambda \mathrm{e}^{-\left(\lambda_{0}+\lambda\right) x}, \\
& n_{a a}\left(x, 0^{+}\right)=\lambda^{2} S_{T} \mathrm{e}^{-\left(\lambda_{0}+\lambda\right) x},
\end{aligned}
$$

where $\lambda_{0} S_{T}=N .{ }^{9}$ Since these densities are exponential, the system can be solved analytically with the following substitutions in Eqs. (56) and (59): $\lambda$ becomes $\lambda+\lambda_{0} ; N_{a t}(0)$ becomes $2\left(\lambda_{0} S_{T}\right) \lambda /\left(\lambda+\lambda_{0}\right)$; and $N / S_{T}$ becomes $\lambda_{0}$. The results are then

$$
\begin{aligned}
n_{r}(x, \infty) & =\frac{1}{2 x}\left(\mathrm{e}^{-\left(N / S_{T}\right) x}-\mathrm{e}^{-\lambda x}\right) \\
& =\frac{1}{2 x}\left(\mathrm{e}^{-\lambda_{0} x}-\mathrm{e}^{-\left(\lambda+\lambda_{0}\right) x}\right), \\
n_{t t}(x, \infty) & =\frac{N_{a t}(0)}{2} \frac{\lambda\left(N / S_{T}\right)}{\lambda-\left(N / S_{T}\right)}\left(\mathrm{e}^{-\left(N / S_{T}\right) x}-\mathrm{e}^{-\lambda x}\right) \\
& =\lambda_{0}^{2} S_{T}\left(\mathrm{e}^{-\lambda_{0} x}-\mathrm{e}^{-\left(\lambda+\lambda_{0}\right) x}\right),
\end{aligned}
$$

which implies that

$$
\begin{aligned}
n_{\delta}(x, \infty)+n_{t t}(x, \infty) & =n_{\delta}\left(x, 0^{+}\right)+n_{t t}(x, \infty) \\
& =\lambda_{0}^{2} S_{T} \mathrm{e}^{-\left(\lambda_{0}+\lambda\right) x}+\lambda_{0}^{2} S_{T}\left(\mathrm{e}^{-\lambda_{0} x}-\mathrm{e}^{-\left(\lambda+\lambda_{0}\right) x}\right) \\
& =\lambda_{0}^{2} S_{T} \mathrm{e}^{-\lambda_{0} x}=n_{\delta}\left(x, 0^{-}\right) .
\end{aligned}
$$

Calculations using $1 / \lambda_{0}=1 \mathrm{Mb}$ and 80 and $160 \mathrm{~Gy}$ indicate that the ring density is negligible (in any case, rings do not enter pulsed-field gels unless they are small [20]). Eq. (80) thus states that exponentially distributed cut-somes produce final number densities that are indistinguishable from eurejoined densities, i.e. irradiated cells will appear no different from unirradiated cells. To obtain misrejoining information from PFGE experiments conducted at large times, the model thus suggests that cutters should be as non-random as possible, the extreme being that all fragments have the same size.

For a non-random cut-some distribution, suppose $n_{\delta}\left(x, 0^{-}\right)$is Gaussian with mean $\mu=3.2 \mathrm{Mb}$ and standard deviation $\sigma=0.3 \mathrm{Mb}$, where we chose the mean to match a particularly important cut-some [2]. Applying Eqs. (68)-(70) numer-

\footnotetext{
${ }^{9}$ The sum of these initial densities equals $\left[\left(\lambda_{0}+\lambda\right) S_{T}\right]\left(\lambda_{0}+\lambda\right) \mathrm{e}^{-\left(\lambda_{0}+\lambda\right) x}$, i.e. the original cut-some distribution has a dose equivalent which adds to the external dose.
} 
ically to this cut-some density yields an initial state $\left\{n_{a t}\left(x, 0^{+}\right), n_{a a}\left(x, 0^{+}\right)\right.$, $\left.n_{\delta}\left(x, 0^{+}\right), 0,0\right\}$ as a set of three vectors (we used $\Delta x=0.01$ and vector lengths of 1024). The steps outlined in Section 7.2 were then used to compute the SSEB final state $\left\{0,0, n_{\delta}(x, \infty), n_{r}(x, \infty), n_{t t}(x, \infty)\right\}$ where $n_{\delta}(x, \infty)=n_{\delta}\left(x, 0^{+}\right)$. The sums of these initial and final densities are plotted in Fig. 6(a) and (b), respectively.

Suppose instead that the cut-some distribution is broadened to a Gaussian with $\mu=3.2 \mathrm{Mb}$ and $\sigma=2 \mathrm{Mb}$. We then obtain the final number densities shown in Fig. 7. Compared to Fig. 6 we see that broader (and thus more realistic) cut-some distributions manifest their misrejoinings through peak shifts to smaller fragment sizes, rather than decrements in peak height as shown in Fig. 6 for narrow bands. For this situation an approach to PFGE measurements analogous to Dietzel's flow karyotype approach would break down because peak (unperturbed or eurejoined) and background (misrejoined) signals can no longer be separated by inspection. The SSEB model, however, can still be used because it only requires that the sum of the signals be known. Thus a fit of $n_{\delta}\left(x, 0^{+}\right)+n_{t t}(x, \infty)$ to observed final densities will yield distribution-based estimates of $\lambda$, the expected number of misrejoinings per base pair.

\section{Discussion}

\subsection{Mathematical results}

Time integrals of the non-linear integro-differential SSEB model were obtained using Fourier transform techniques. For low LET doses high enough to satisfy both high-dose conditions (Section 6), closed form analytic solutions showed us that: (1) edges and internals initially exponential, remain exponential, their final state mean values approaching the average chromosome size; and (2) in the limit of very high-doses, the final ring mass approaches one-half the average chromosome mass. At realistic doses the exact form of the EtBr model was used as the initial state of the SSEB model and numerical solutions were obtained using very efficient FFT methods.

\subsection{Rings}

The results on rings show that one of the model idealizations, neglecting ring source terms in Eq. (5), is appropriate for the doses of interest. For mammalian chromosomes at doses between 1 and 5 Gy (cut-some doses can be much higher), the yield of rings is predicted to be a constant times the bitelomere yield, the proportionality constant depending on the distribution of chromosome sizes as well as the localization of interphase chromosomes to nuclear subdomains. These results on rings are effectively identical to results obtained by a very different approach using Monte Carlo simulations [17]. 

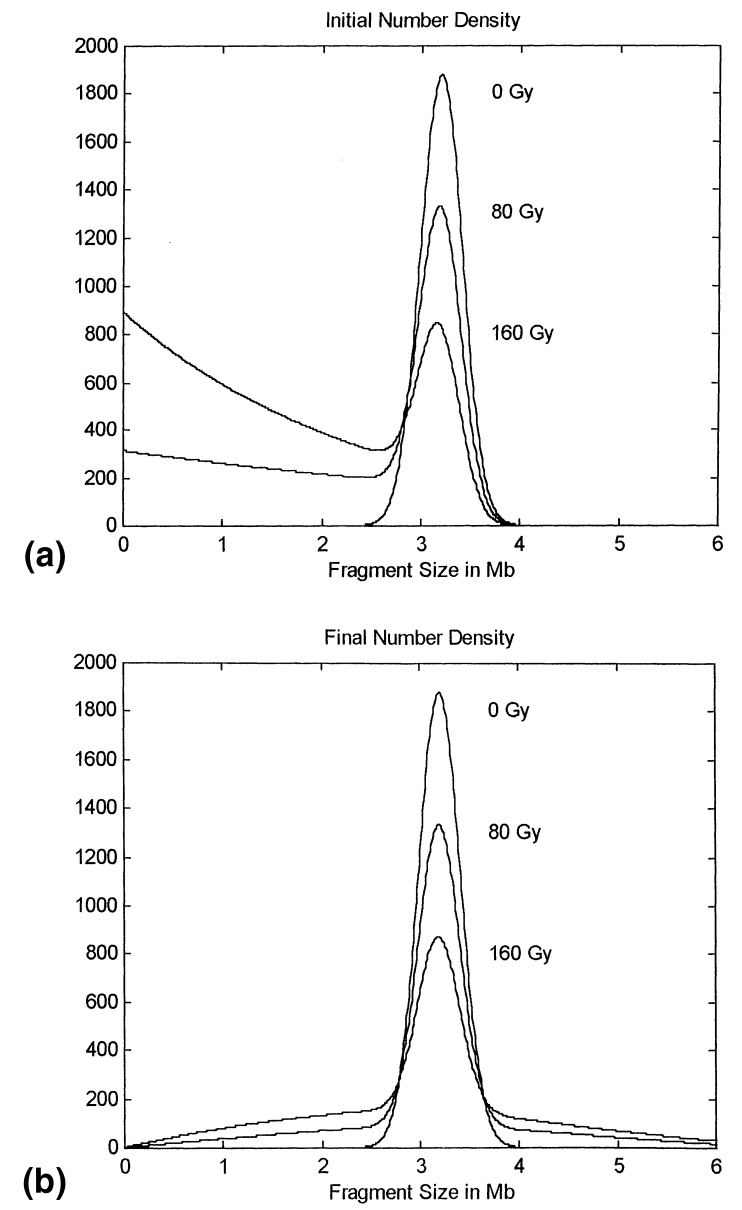

Fig. 6. Gaussian distributed ( $\mu=3.2 \mathrm{Mb}, \sigma=0.3 \mathrm{Mb}$ ) cut-somes at $t=0^{-}$were converted to SSEB initial conditions at $t=0^{+}$(a) using the continuum form of the EtBr model (Eqs. (68)-(70)) with doses of 0,80 and $160 \mathrm{~Gy}$. In the corresponding final densities (b) misrejoining results in peak reduction and increases in background.

\subsection{Flow karyotype assays}

In Section 7.2 we examined potential SSEB applications to flow karyotype data in the range of 5-50 Gy. We suggest that such data could be informative about DSB rejoining mechanisms. Given such data, the SSEB model could be applied to form distribution-based estimates of the total misrejoinings. One advantage SSEB has over Dietzel's PRA and BNA methods [10] is that it does not assume that only a negligible number of chromosomes have more than one 


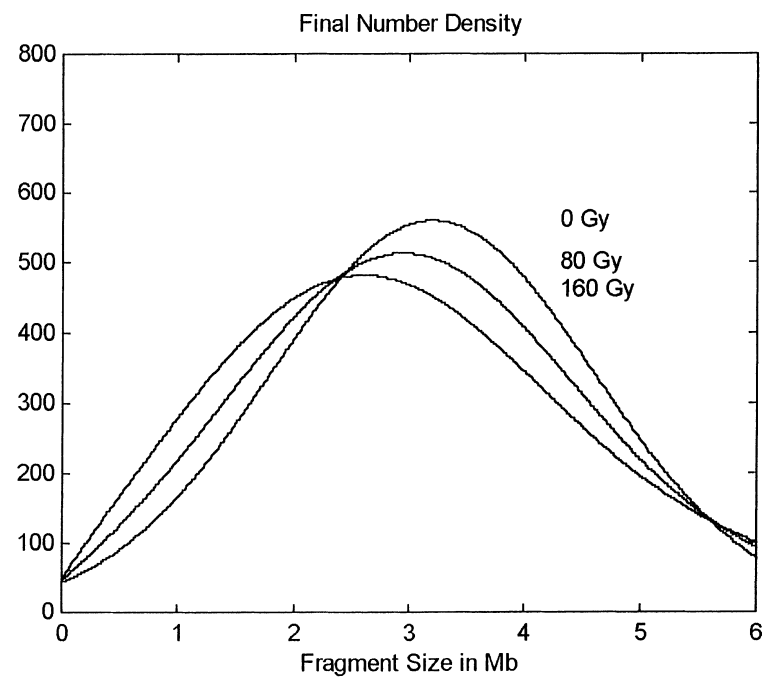

Fig. 7. For a Gaussian cut-some distribution with $\mu=3.2 \mathrm{Mb}$ and $\sigma=2.0 \mathrm{Mb}$, misrejoining manifests itself mostly through shifts in the final density toward smaller $x$. Compare this to the right panel of Fig. 6 where peak reduction dominates the dose-response.

misrejoining, an assumption that limits Dietzel's algorithms to doses less that 10 Gy if human chromosomes are used (Table 1).

The utility of the SSEB approach is seen quite clearly for doses on the order of 50 Gy (Fig. 5(b)bottom) where all the peaks have vanished and the number of misrejoined fragments stays roughly constant with dose (it saturates at the number of chromosomes). In this case PRA breaks down because there are no peaks and BNA breaks down because the total background between the peaks no longer changes with increments in dose. Meanwhile, shape changes in the misrejoined distribution can still be detected, the maximum of the density continuing to move toward smaller fragment sizes as dose increases. The SSEB model can capture these changes in the form of misrejoining estimates. Note that as the high dose exponential limit is approached, SSEB fails to detect any further misrejoining because there are no changes in the final density with increments in dose (unless ring formation kicks in), at lower doses Dietzel's assumptions apply and, at least for chromosomes, the advantages of SSEB diminish with decreasing dose.

\subsection{PFGE data}

For cut-somes, the assumption that two or more DSBs on one cut-some are improbable will be valid to high doses, but there is a different limitation for an approach analogous to that of Dietzel et al.: a very restrictive assumption that 
cut-some sizes have a peak pattern as in Fig. 6. This limitation is relaxed with the SSEB approach because the separability of eurejoined and misrejoined signals is not required - SSEB fits to their sum while PRA and BNA requires each of the signals separately. The SSEB approach is thus more robust with respect to the amount of tolerance it has for randomization in the cut-some distribution. Overall, with low levels of randomization (Fig. 6) both methods can derive misrejoining estimates, at intermediate levels (Fig. 7) only the SSEB approach can estimate misrejoining, and when complete randomness is reached (exponentially distributed cut-somes) both methods fail.

Since cut-somes are much smaller than chromosomes, very high doses (over 80 Gy [2]) are needed to create readily detectable damage. At such doses, cellular DSB rejoining mechanisms may change for any of several reasons, a few of which may be: altered chromatin structure, saturated rejoining enzymes, changing redox state, induced apoptosis, etc. One must therefore be cautious when applying models based on cut-some data to lower doses.

\subsection{Summary}

It is possible to predict systematically the shape of size distributions for misrejoining DNA fragments. This size information is available in many experiments and the theoretical curves given here should improve numerical estimates of such basic quantities as the total number of misrejoinings at a given dose. This is the kind of quantitative information needed for understanding DSB misrejoining and, ultimately, for extrapolating laboratory data on DSBs to carcinogenesis risk estimates.

\section{Acknowledgements}

We are grateful to B. Rydberg for discussions. T.R. and D.G.H. were supported by the United States Department of Energy under grant DE-FG0192EW50625, P.H. by NSF grant BIR-963-0735, and R.K.S. by NSF grant DMS-9532055.

\section{References}

[1] M.N. Cornforth, Radiation-induced damage and the formation of chromosome aberrations, in: J.A. Nickoloff, M.F. Hoekstra (Eds.), DNA Damage and Repair - Biochemistry, Genetics, and Cell Biology, Humana Press, NJ, 1997.

[2] M. Löbrich, B. Rydberg, P.K. Cooper, Repair of X-ray induced DNA double-strand breaks in specific Not I restriction fragments in human fibroblasts: Joining of correct and incorrect ends, Proc. Natl. Acad. Sci. 92 (1995) 12050. 
[3] C.R. Contopoulou, V.E. Cook, R.K. Mortimer, Analysis of DNA double strand breakage and repair using orthogonal field alternation gel electrophoresis, Yeast 3 (1987) 71.

[4] D. Blöcher, In CHEF electrophoresis a linear induction of DSB corresponds to a non-linear fraction of extracted DNA with dose, Int. J. Radiat. Biol. 57 (1990) 7.

[5] V.E. Cook, R.K. Mortimer, A quantitative model of DNA fragments generated by ionizing radiation, and possible experimental applications, Radiat. Res. 125 (1991) 102.

[6] T. Radivoyevitch, B. Cedervall, Mathematical analysis of DNA fragment distribution models used with pulsed-field gel electrophoresis, Electrophoresis 17 (1996) 1087.

[7] T. Radivoyevitch, Mathematical Models of Misrejoining DNA Double-Strand Breaks and DNA Fragment Size Distributions, PhD thesis, Department of Biometry and Epidemiology, Medical Univeristy of South Carolina, Charleston, SC 29425, December 1996.

[8] B. Cedervall, P. Källman, W.C. Dewey, Repair of DNA double-strand breaks: Errors encountered in the determination of half-life times in pulsed-field gel electrophoresis and neutral filter elution, Radiat. Res. 142 (1995) 23.

[9] A. Kraxenberger, A.A. Friedl, A.M. Kellerer, Computer simulation of pulsed-field gel runs allows the quantitation of radiation-induced double-strand breaks in yeast, Electrophoresis 15 (1994) 128.

[10] A.H. Dietzel, J. Hain, R.P. Virsik-Peuckert, D. Harder, Algorithms for evaluation of radiation induced chromosome aberration yields per cell from flow karyotypes, Cytometry 11 (1990) 708.

[11] K. Sax, An analysis of X-ray induced chromosomal aberrations in tradescantia, Genetics 25 (1940) 41.

[12] T. Radivoyevitch, D.G. Hoel, A.M. Chen, R.K. Sachs, Misrejoining of double-strand breaks after X-irradiation: Relating moderate to very high doses by a Markov model, Radiat. Res. 149 (1998) 59.

[13] M. Löbrich, P.K. Cooper, B. Rydberg, Non-random distribution of DNA double-strand breaks induced by particle irradiation, Int. J. Radiat. Biol. 70 (1996) 493.

[14] H.C. Newman, K.M. Prise, M. Folkard, B.D. Michael, DNA double-strand break distributions in X-ray and alpha-particle irradiated V79 cells: evidence for non-random breakage, Int. J. Radiat. Biol. 71 (1997) 347.

[15] A.V. Oppenheim, A.S. Wilsky, I.T. Young, Signals and Systems, Prentice-Hall, Englewood Cliffs, NJ, 1983.

[16] L. Hlatky, R.K. Sachs, P. Hahnfeldt, The ratio of centric rings to dicentrics in irradiated human lymphocytes, Radiat. Res. 129 (1992) 304.

[17] A.M. Chen, J.N. Lucas, F.S. Hill, D.J. Brenner, R.K. Sachs, Proximity effects for chromosome aberrations measured by FISH, Int. J. Radiat. Biol. 69 (1996) 411.

[18] M. Löbrich, S. Ikpeme, J. Kiefer, Measurement of DNA double-strand breaks in mammalian cells by pulsed-field gel electrophoresis: A new approach using rarely cutting restriction enzymes, Radiat. Res. 138 (1994) 186.

[19] A.A. Friedl, A. Kraxenberger, F. Eckardt-Schupp, An electrophoretic approach to the assessment of the spatial distribution of DNA double-strand breaks in mammalian cells, Electrophoresis 16 (1995) 1865.

[20] S.D. Levene, B.H. Zimm, Separations of open circular DNA using pulsed-field electrophoresis, Proc. Natl. Acad. Sci. 84 (1987) 4054. 